\title{
Tactical Satellite 3 CDL COMMUNICATIONS, A COMMUNICATIONS LINK FOR MISSION UTILITY
}

\author{
Richard Galindez, \\ L-3 Communications, CSW \\ Salt Lake City, UT \\ CDL Payload Project Engineer \\ 801-594-3124 \\ Richard.Galindez@L-3com.com \\ Thom Davis, \\ AFRL-VSE \\ Kirtland AFB, NM \\ TacSat-3 Program Manager \\ 505-846-5754 \\ Thom.Davis@kirtland.af.mil
}

\begin{abstract}
$\underline{\text { Abstract }}$
Tactical Satellites (TacSats) are part of the Operational Responsive Space (ORS) demonstration program to develop rapid response, low cost, small satellites, whose payloads support real-time data delivery to the combatant commander. As each TacSat is developed, further knowledge is gained towards the end operational systems.

Common Data Link (CDL) is the US military's standard wideband communications waveform for Intelligence Surveillance \& Reconnaissance (ISR) in airborne platforms. In supporting this standard, the military has numerous air, sea and ground CDL systems for theater connectivity.

While CDL is the standard for airborne ISR, it was not implemented in space until TacSat-2 launched on December 16, 2006. TacSat-2's CDL payload supports a 274 Mbps downlink and a 200 Kbps uplink. A CDL system in space brings tactical ISR data directly into existing theater ground stations, allowing for responsive tasking and collection.

Late in 2007, another CDL communications payload will be launched on TacSat-3. TacSat-3 takes the CDL communications payload a step further with networking capability and multiple data rates to continue demonstrating direct theater tasking, collection and dissemination. The satellite will support a 274 Mbps downlink data rate in addition to lower data rates for potential Remote Operated Video Enhanced Receiver (ROVER) connectivity. Rover III is a portable receive-only terminal that displays sensor data from multiple airborne platforms across $\mathrm{Ku}, \mathrm{C}$, and L-Band with over 1000 units in use.
\end{abstract}

Each CDL payload delivered was based on utilizing an existing airborne design. This paper will focus on the challenges of modifying the CDL airborne system for the TacSat-3 launch and space environment while discussing the enhanced mission operation utility CDL provides to the combatant commander.

\section{Communication Payload Introduction}

With the successful delivery and launch of the TacSat-2 CDL communications payload, L-3 Communications, CSW was placed under contract by Air Force Research Laboratory (AFRL VSE and IFGD) to deliver another airborne CDL communications system destined for space with a planned delivery date of April 2007. In addition to the Microwave Modem Assembly (MMA) implemented for TacSat-2, L3 developed a Radio Frequency Assembly (RFA) for transmit/receive amplification and two antennas. A 12" parabolic is for duplex operation with the ground station while a broad-beam horn antenna is for Rover operation at a lower data rate. A very basic CDL payload block diagram is shown in Figure 1 with the external spacecraft electrical interfaces shown in grey.
Galindez

22 CFR 125.4(b)(13) applicable \# (07-S-1535)
1 of 5

$21^{\text {st }}$ Annual AIAA/USU

Conference on Small Satellites 


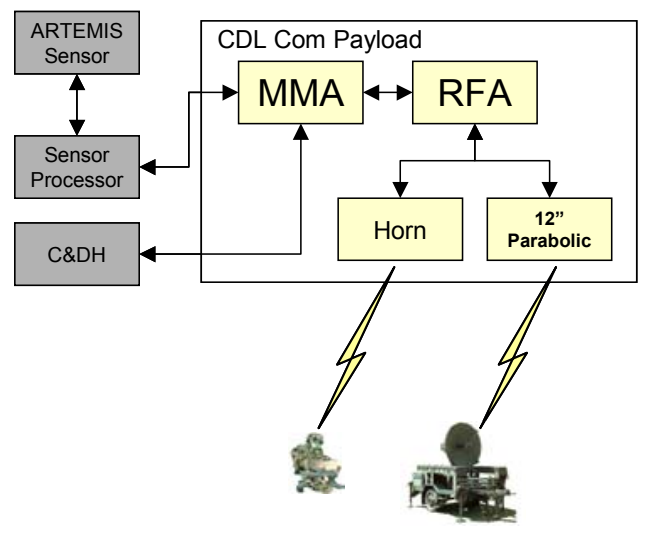

Figure 1 TacSat-3 CDL Communications Payload Block Diagram

L-3 was tasked with a serious challenge with this next version of TacSat in that AFRL wanted an airborne system that would last up to a year in the Low Earth Orbit environment. This type of reliability is not easily given since the Microwave Modem Assembly (MMA) was designed for an F-16 fighter pod.

While the F-16 fighter pod is by no means a benign environment, it pales in comparison to the Minotaur launch and radiation in the cold vacuum of space. Not only is the space LEO environment cold on the order of $-100^{\circ} \mathrm{C}$, but temperature swings up to $+100^{\circ} \mathrm{C}$ are possible due to solar heating. (For reference space with no heating or light around is $-2.7 \mathrm{~K}$, or $-270^{\circ} \mathrm{C}$ ) Even Mil-Standard components just don't cope with that type of environment without some type of assistance. Needless to say L-3, CSW had their work cut out for them.

\section{Airborne System for Space?}

Now one may ask, why would the Government want to go through such a difficult task of having L-3, CSW force an airborne based communications system into a space environment? Not only is the space environment harsher than the airborne environment, but Size, Weight, and Power (SWAP) are far more critical when you factor in the spacecraft design impact in relationship to the launch vehicle. A similar space qualified modem (modulator - demodulator) design using rad hard components would be roughly half the size, weight and power of an airborne modem system. L-3, CSW is aware of the difficulty, having started development of such a system back in 2003. Table 1 represents the differences between the space qualified and the airborne design.
Table 1. Size weight and power for the space qualified system represents a significant savings over a similar airborne system. ${ }^{1}$

\begin{tabular}{|c|c|c|c|}
\hline $\begin{array}{c}\text { Similar Airborne } \\
\text { CDL System }\end{array}$ & $\begin{array}{c}\text { Dimensions } \\
\text { (inches) }\end{array}$ & $\begin{array}{c}\text { Weight } \\
\text { (pounds) }\end{array}$ & $\begin{array}{c}\text { Power } \\
\text { (W) }\end{array}$ \\
\hline Modem Section & $15.75 \times 11 \times 11.18$ & 41.6 & 188 \\
\hline TOTAL & 1938 cu-in & $41.6 \mathrm{lb}$ & $188.0 \mathrm{~W}$ \\
\hline $\begin{array}{c}\text { Space Qualified } \\
\text { CDL System }\end{array}$ & $\begin{array}{c}\text { Dimensions } \\
\text { (inches) }\end{array}$ & $\begin{array}{c}\text { Weight } \\
\text { (pounds) }\end{array}$ & $\begin{array}{c}\text { Power } \\
\text { (W) }\end{array}$ \\
\hline \begin{tabular}{c} 
Downlink Stack \\
\hline $\begin{array}{c}\text { Uplink/COMSEC } \\
\text { Stack }\end{array}$
\end{tabular} & $8.5 \times 8.5 \times 2.88$ & 9.0 & 25.2 \\
\hline TOTAL & $518.8 \mathrm{cu}$-in & $22.5 \mathrm{lb}$ & $79.0 \mathrm{~W}$ \\
\hline
\end{tabular}

The problem as is seemingly always the case was time and money. AFRL simply did not have the time or money for L-3, CSW to complete the space qualified design. The TacSat programs have created a new breed of satellite. These satellites are procured quickly and with minimal cost.

\section{Operational Responsive Space Paradigm}

Tactical Satellites (TacSats) are part of the Operational Responsive Space (ORS) demonstration program to develop rapid response, low cost, small satellites, whose payloads support real-time data delivery to the combatant commander. As each TacSat is developed, further knowledge is gained towards the end operational systems.

Bottom line, if one waits for things to be done perfectly you may never get to where you want to go. Taking risk and meeting a lower threshold of typical requirements drives down cost and schedule to the point that you can launch a satellite from design to orbit in under 2 years. Traditional satellite systems take 5-7 yrs with costs in the 100's of millions if one is lucky, not to mention a 60-100 million dollar launch cost that is approximately ten times greater than a small sat launch. When one looks at designing, building and launching a small sat for less than the traditional satellites' launch costs alone, one can start to see how responsive TacSats can become.

\section{Why CDL in Space?}

So now that we have a glimpse into the paradigm of doing things faster and taking some risk, one might ask why use the Common Data Link (CDL) in space to begin with. There are two parts to that answer; 1) existing infrastructure in theater and 2) lower lifetime costs. 
The existing infrastructure answer requires a little background on CDL to explain. CDL is the US military's standard wideband communications waveform for Intelligence Surveillance \& Reconnaissance (ISR) in airborne platforms. In supporting this standard, the military has numerous air, sea and ground CDL systems for theater connectivity. Specifically the existing ground stations can be timeshared for the TacSat mission. What better way to get real-time data to the combatant commander than to leverage his existing systems that his men are already familiar with.

The second part of the answer almost falls out of the first answer. Since existing infrastructure is being used for the TacSat mission downlink, the military does not have to worry about dragging out more equipment into theater. The combatant commander's troops can use the same ground station they use for airborne ISR missions as satellite missions. Today this is being done for TacSat-2. An existing airborne ISR ground station is being shared with the TacSat- 2 mission payload communications. Remember that TacSat-2 and 3 are Low Earth Orbit (LEO) satellites and because of their orbit, they only have ground access six times a day with each pass less than 10 minutes. That type of orbit lends itself well for mission sharing with the airborne assets. A potential mission configuration is depicted in Figure $\underline{\mathbf{2}}$ below.

\section{Figure 2 Notional TacSat-3 Tactical CONOPS ${ }^{2}$}

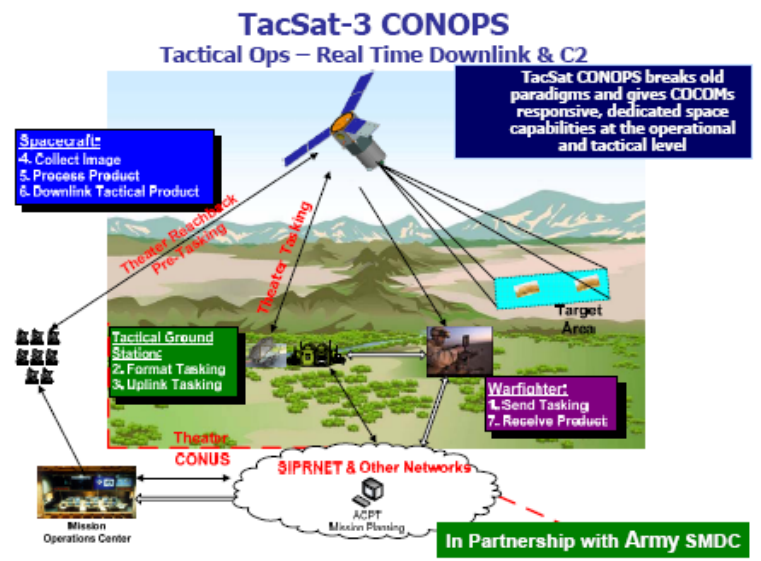

Figure 2 shows the sequence of events for the TacSat- 3 tactical CONOPS. The sequence starts with the warfighter sending a collection task to the CDL ground station prior to a collection opportunity. A collection opportunity is defined as any time TacSat-3 can collect a Hyperspectral image within the assigned theater. The CDL ground station uplinks the formatted tasking data to the satellite. The tasking can be modified at any moment until after a short period of time after acquisition of signal with the CDL ground station.

Upon receipt of the properly formatted tasking, TacSat3 autonomously slews to the target, collects the data, processes the data, then downlinks the data directly to the warfighter. The tactical data product is determined by the original tasking, and is tailorable to meet the warfighter's needs and communications capabilities. TacSat-3's objective is to demonstrate in theater, the tasking, imaging, processing, and direct down link of the HSI data in less than 10 minutes.

Once the tactical product has been disseminated in theater, the raw data is downlinked to the next available site. The raw data can be further processed for more detailed products.

\section{Back to the Challenge - Peeling the Onion}

Now that you the reader has some background on

- harsh space environment

- why one would want to use an airborne system in space

- ORS concept

- and lastly why CDL in space, we can get down to the challenge at hand: assuring AFRL that the airborne system will work in space up to a year. With the support of Aerospace Corp. and two additional consultants, L3 started to pick the system apart piece by piece to evaluate against the environment. L3 started generating parts lists of the existing CDL system to evaluate the following aspects:

- metals

- outgassing

- thermal

- mechanical

- radiation

Parts

The L3 Communications system is comprised of an airborne Microwave Modem Assembly (MMA), Radio Frequency Assembly (RFA), 12" parabolic antenna, and a broadbeam horn. The RFA and antenna's were relatively easy since they would be new designs which could be built specifically for the space environment, but the MMA was an existing airborne design and major modifications could not be made due to schedule and funding limitations. The MMA is a card cage chassis comprised of many slide in SEM-E modules that perform various functions:

- network processing

- $\quad$ system processing

- multiplexing/demultiplexing

- encryption 
- forward error correction (fec)

- modulation/demodulation

- timing

- up/down conversion

- filtering

- power conversion

With the functions the MMA performs there are $>12,000$ parts. Of those 12,000 , there are around 1,000 active parts, with around 350 that are unique.

\section{Metals}

L3 reviewed their entire parts list for pure tin, zinc, and cadmium since they could not be used based on whisker and sublimation concerns. Pure tin and zinc were not a big problem but many connectors in the airborne design were cadmium plated to minimize salt corrosion. They had to find replacement connector parts and modify existing documentation to get these items in house for the build. The tricky part was getting these items in time for the build since they were mostly long lead items.

\section{Outgassing}

They also had to minimize the systems outgassing to the greatest extent we could and used the standard Total Mass Loss (TML) $<1.0 \%$ and Collected Volatile Condensable Materials $(\mathrm{CVCM})<0.1 \%$ limits. NASA's Outgassing website: http://outgassing.nasa.gov/ was extremely useful in evaluating the various materials used in the current L3 system although on several occasions they had to go to the vendor to determine the chemical properties. Thermal compounds, adhesives/epoxies, potting, conformal coatings, wire insulation, cable tie downs, and paints all came under scrutiny and under most circumstances required replacements and build documentation modifications.

\section{Thermal}

Thermally, the MMA and RFA are maintained by the spacecraft bus with a cold plate that is strangely enough more benign than our normal airborne environment. The same could not be said for our parabolic antenna since it is isolated from the bus and would see $\pm 100^{\circ} \mathrm{C}$ temperature swings. Some unique antenna designs were developed to endure the temperature range.

\section{Mechanical}

Mechanically, the Microwave Modem Assembly did not require modifications based on the soft-ride data received for the launch vehicle. Although a fair amount of analysis had to be run to verify those conditions since the input vibration profile is significantly different than a typical airborne platform.

\section{Radiation}

One of the key space environmental conditions L3 had to overcome was radiation. While TacSat- 2 continues to operate in a similar environment, that cannot be used as a basis for the TacSat-3 system. This is especially true since L3 Communications had the additional networking functionality parts to consider.

Through analysis of the orbit and inclination angle over the mission duration L3 was able to determine that the Total Ionizing Dose (TID) would be $<70 \mathrm{rad}$ (Si). This amount of dosage is insignificant to most electronics and is not considered a significant risk.

Single Event Effects (SEE) are not as easy to analyze for as TID, since test data is not available to evaluate for all the CDL parts. Aerospace reviewed most of the active parts and came up with 44 that had an unknown risk. Eventually data was found on 5 of the 44 parts that reduced some risk, but that still left 39 parts with an unquantifiable risk. L3 then searched for radiation hardened replacements that were pin-for-pin compatible but none existed. Luck would come when an orbit change was made for the imager that lowered our inclination angle from $60^{\circ}$ to $41^{\circ}$ that significantly reduced the high-energy particle fluence. The orbit comparisons are depicted in Figure 3 below.

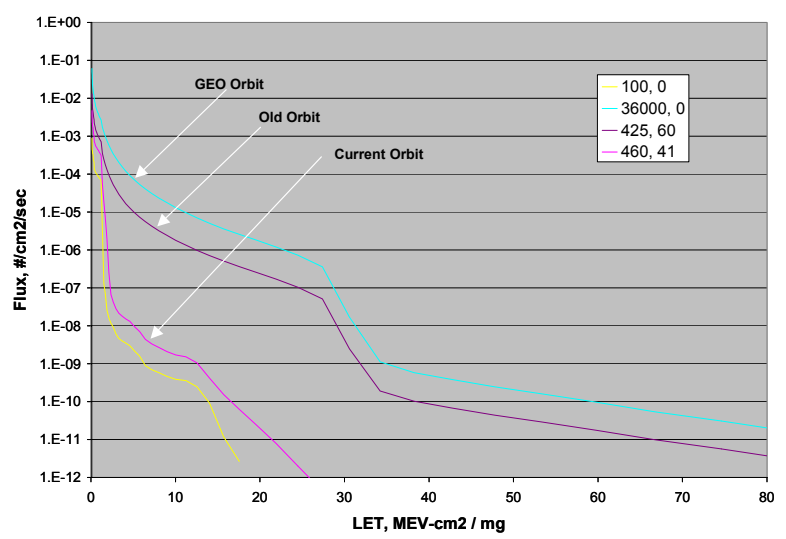

Figure 3. Flux vs LET for Various Orbits

This reduction in high energy particles along with the system only being on around 45 minutes/day and not being on while over the South Atlantic Anomaly (SAA) greatly increases our probability of success over the year mission duration. For reference, Single Event Effects (SEE) caused by high energy particles do not occur when equipment is powered down. Also for reference, the SAA is a region around Brazil as low as $200-300 \mathrm{Km}$ that has a significantly higher energy proton flux $(>10 \mathrm{MeV}$ at a typical flux of 3000 particles per square centimeter per second). ${ }^{3}$ 


\section{Summary}

TacSat-3 is a responsive space mission with a focus to provide a militarily relevant capability to the warfighter, while meeting the cost and schedule aspects of the Operationally Responsive Space paradigm. Utilizing CDL in space provides the benefit of using the existing in-theater CDL ground infrastructure for tactical communications. Many challenges have been overcome in the development of a modified CDL airborne system for space. While there is no way to drive risk to zero within the schedule and funding available, the mission success probability has greatly increased through analysis and other efforts. Additionally, each day as the TacSat-2 CDL airborne system operates in space, the risk is lowered even more. While the efforts presented for the airborne system in space have met the current program needs, by no way is this a preferred approach. The best way forward for future programs is to complete the CDL space qualified system. This approach provides the least impact to a program in terms of its size, weight, and power as referenced in Table 1. This approach also uses radiation hardened components and design methodologies that drive the Single Event Effects (SEE) risk to near zero. Lastly, the space qualified system is designed from the outset to meet the harsh launch and space environment.

\section{Additional CDL Background $^{4}$}

In 1979, the Common Data Link (CDL) Program foundation originated the Interoperable Data Link (IDL) program. The United States Air Force/Assistant Secretary of Defense (USAF/ASD) and the National Security Agency (NSA) developed the IDL U-2 platform. In 1988, the Office of the ASD (OASD)/Command, Control, Communications, and Intelligence (C3I) recognized the success of the IDL program with a decision to develop a standard communication architecture that would be common across all Department of Defense (DoD) Services. $\mathrm{OASD} / \mathrm{C} 3 \mathrm{I}$ mandated the $\mathrm{CDL}$ proliferation to users involved in the collection and dissemination of wideband Intelligence, Surveillance, and Reconnaissance (ISR) data.

CDL is a full-duplex, jam resistant spread spectrum, point-to-point digital link. The uplink operates at $200 \mathrm{kbps}$-and possibly up to $45 \mathrm{Mbps}$. The downlink can operate at $10.71-45 \mathrm{Mbps}, 137 \mathrm{Mbps}$, or $274 \mathrm{Mbps}$. In addition; future rates of $548 \mathrm{Mbps}$ and $1096 \mathrm{Mbps}$ can potentially be supported.

The Common Data Link program is designed to achieve data link interoperability and provide seamless communications between multiple Intelligence, Surveillance, and Reconnaissance (ISR) collection systems operated by armed services and government agencies. CDL provides full-duplex, jam resistant, digital microwave communications between the ISR sensor, sensor platform, and surface terminals. The CDL Program establishes data link standards and specifications identifying compatibility and interoperability requirements between collection platforms and surface terminals across user organizations.

\section{References}

1. Galindez, Richard, et al. "A Common Data Link (CDL) for Space-Based Communications: Migration of Airborne Hardware to Space", Small Satellite Conference, Aug. 2005

2. Davis, Thomas M. and Capt. Stanley D. Straight, "Development of the Tactical Satellite 3 for Responsive Space Mission"s, Responsive Space Conference, Apr. 2006

3. Spera, Gabriel, "A Space Oddity”, Crosslink Summer 2003, Aerospace Corp, http://www.aero.org/publications/crosslink/su mmer2003/backpage.html

4. Joint Interoperability Test Command, http://jitc.fhu.disa.mil/cdl/desc.html $21^{\text {st }}$ Annual AIAA/USU

Conference on Small Satellites 